\title{
2006-1396: TEAM WORK EXPERIENCES IN PROCESS AUTOMATION FOR PRECISION CHEMICAL DEPOSITION
}

\section{Jeffrey Evans, Purdue University}

Jeffrey J. Evans is an Assistant Professor of Electrical and Computer Engineering Technology at Purdue University, West Lafayette, Indiana. He has a B.S. in Electrical Engineering Technology from Purdue University, and M.S. and Ph.D. degrees in Computer Science from the Illinois Institute of Technology in Chicago, Illinois. Prior to joining Purdue he held engineering positions over a 20-year career developing hardware, software, and systems in several industries including automotive control systems, consumer and industrial warning and safety, medical, and telecommunications systems. He is a member of the ASEE, ACM, and a senior member of the IEEE. His research interests include understanding the mechanisms that create performance degradation in distributed computing systems, focusing in the areas of high performance computing and sensor networks.

\section{Matthew Kirleis, Purdue University}

Matt Kirleis is a senior in the Electrical and Computer Engineering Technology program at Purdue University in West Lafayette, Indiana. His academic interests include microcontrollers, communications and controls. He is a member of Tau Alpha Pi. In his spare time he enjoys working on various electrical projects such as building an RC Engine Dynamometer for his senior project.

\section{Casey Smith, Purdue University}

Casey Smith earned his bachelors degree in Electrical Engineering Technology at Purdue University, West Lafayette, Indiana, in December 2005. His academic interests included analog circuitry, programming and project development. He is currently employed at Advanced Micro Devices in Austin, Texas, where he performs thermal analysis for customer support engineering. 


\title{
Teamwork Experiences in Process Automation for Precision Chemical Deposition
}

\begin{abstract}
The area of chemical analysis is growing rapidly, supporting areas related to Bioengineering, such as pharmaceuticals and genetics. High throughput of analyses is becoming more critical. As the size of each sample shrinks, so to does the time available to perform accurate analysis due to evaporation and other contamination factors. New materials and processes are being created in order to provide the ability to analyze large numbers of samples in a reasonable period of time. One such project at Purdue is reducing the size of samples even further, while increasing the spatial density of a group of samples. This requires increased precision in two-dimensional alignment and sample deposition.
\end{abstract}

Students in electrical and computer engineering technology participated in an interdisciplinary project with chemical engineering students to automate a mostly manual alignment and deposition process. Automation of this process involves the integration and precise computer control of several disjoint instruments. The primary objective of this work was to increase throughput by several orders of magnitude. Secondary goals include system characterization and prediction of throughput upper bounds using available technology and off-the-shelf components. Successful completion of these objectives requires a combination of skills learned in the classroom as well as teamwork among students within specific disciplines as well as those from others, where technical languages and dialects are different.

This paper describes the problem and subsequent design, implementation and system characterization activities undertaken. New and innovative test and measurement techniques were developed, requiring creative solutions using and stretching those learned in the classroom. Descriptions of the obstacles, methods, lessons learned, and throughput improvement results are presented. This work has provided a unique opportunity for interdisciplinary collaboration and research and development experiences for undergraduate students.

\section{Introduction}

The area of chemical analysis is growing rapidly, supporting areas related to Bio-engineering such as pharmaceuticals and genetics. High throughput of analyses is becoming more crit- 
ical. As the size of each sample shrinks, so to does the time available to perform accurate analysis due to evaporation and other contamination factors. New materials and processes are being created in order to provide the ability to analyze large numbers of samples in a reasonable period of time. One such project is reducing the size of samples even further, while increasing the spatial density of a group of samples. This requires increased precision in two-dimensional alignment and sample deposition.

Protein deposition and drug characterization has generally been a tedious and slow manual operation, particularly when large numbers of samples must be evaluated. The proteins themselves are costly, thus the process can be expensive due to the amount of chemical deposited and the time taken to manually deposit, move, and acquire the necessary data. Variables such as chemical evaporation and contamination can bias or even ruin the characterization of a drug due to overexposure to the test environment.

Chemical Engineering researchers at Purdue University recently developed a platform for asymmetric biochemical sensing. It is comprised of an array of micron size "reactors" on a mesoporous alumina membrane integrated with a polymeric layer. This configuration maintains the integrity of the membrane surface and permeability, resulting in a platform for asymmetric biochemical sensing [2]. The device can be used to control enzyme reactions at the surface of the membrane. The small size of the reactors enable new sensing modes, improved stability of biomolecular systems, enhanced sensitivity, and higher throughput.

The goal of this project is to provide researchers in Biology and other related disciplines, and eventually the pharmaceutical industry with an automated platform to enable the evaluation of hundreds, thousands, or more protein samples with speed, accuracy, and repeatability improvements orders of magnitude superior to manual techniques. Using a much smaller, testable sample promises to save the industry time and economic cost by capitalizing on using the same volume of chemical distributed over a larger number of tests.

To accomplish this goal, researchers, graduate students, and undergraduate students were required to work as a team, from requirements generation to user documentation. Undergraduate technology students were required to develop requirements, characterize subsystems, implement solutions, and test and verify the integrated system working in an unfamiliar scientific domain. This combination of tasks and team interactions across disparate scientific disciplines is not common in Technology curriculums. Thus a secondary goal was to observe and document teamwork experiences of this interdisciplinary group.

The remainder of this paper is as follows. Section 2 describes the problem, the formulation of the team, the enabling technology used to address the problem, and a brief description of current testing and analysis methods. The design and methods used to address the automation problem, including team member interactions are presented in section 3. Finally, a summary is provided in section 4, along with observations pertaining to the lessons learned and educational advantages of participating in similar interdisciplinary projects. 


\section{Background}

The process of drug discovery and testing remains for the most part labor intensive. For example, a volume of protein material is generally extracted from a relatively large container manually by a lab technician using a syringe or pipette. A portion of this volume is then inserted into a test tube and placed into a storage element, awaiting the addition of a "reagent", such as the drug under test. Once the reagent is introduced, some reaction occurs (or does not). Data from some sort of "sensor" must be collected to be analyzed later. This process is repeated many fold, using the same then different proteins and reagents.

The materials used for this testing are expensive, and the reactions are slow due to the volumes used for each test. Therefore it would be advantageous to reduce the per test volume of material used, potentially reducing the reaction time. The benefits of reduced material cost and time are obvious, however other ramifications arise. Since the samples are fluids reducing the volume leads to the challenges of evaporation and contamination. Smaller volumes evaporate faster and are more significantly influenced by other contaminants. Moreover, reaction times decrease to the point where a human cannot perform data collection quickly enough.

"Biosensors are being employed in a large and rapidly increasing number of important medical and environmental applications" [2]. In [2], a process for integrating inorganic membranes in polymetric micorfluidics devices that maintains the surface properties and permeability of the membrane is described in detail. This platform has the advantage of reducing test volumes by many orders of magnitude, providing tremendous cost savings. Instead of a test tube, each reaction occurs in a "well" (figure 1) that is on the order of $10-100 \mu m$ in diameter, containing only nlitres of material.

The manual techniques described above using syringes and pipettes are inappropriate for this platform. Rather, semi-specialized equipment including a precision $\mathrm{x}-\mathrm{y}$ table and a microscope connected to a TV monitor is used to locate each well under a precision hydraulic fluid deposition device to introduce the reagent. In addition, a specialized light source and camera is used to capture images of each reaction over time. This resulting image data is then analyzed to determine the outcome of the test. Each component, or subsystem in this system is in itself automated, involving disparate hardware interfaces and software programs. However these subsystems were not integrated into a cohesive and automated system. The initial process of well location and reagent deposition was primarily a manual one.

To locate and align a well to a deposition device a researcher or technician would employ a joystick to move the $\mathrm{x}-\mathrm{y}$ table. Using the TV monitor connected to the microscope a well would be visually aligned with the deposition device. The technician then would interface 


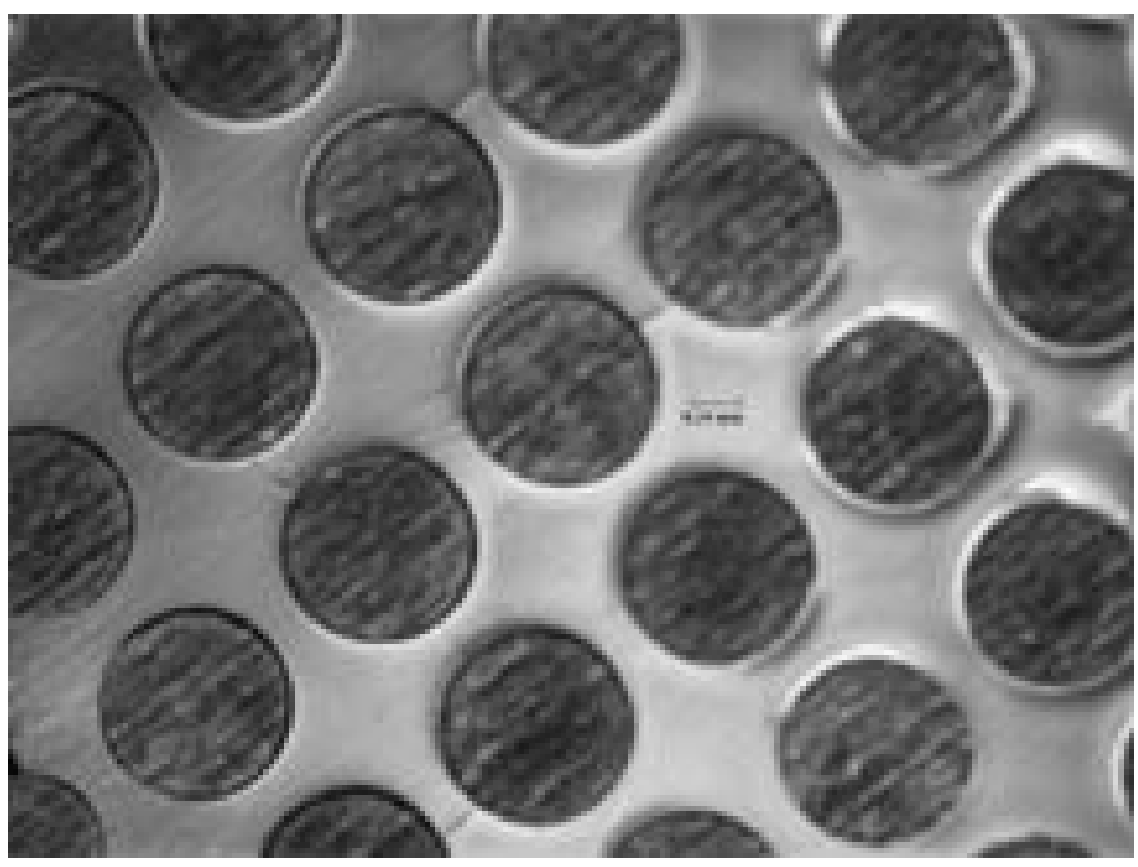

Figure 1: Micro-reactor Platform [1]

with a computer program to deposit the reagent. Once the reagent is introduced, the process is repeated until such a time where reactions were evident or evaporation was of concern. An experienced lab technician can setup about 100 wells, each with between 1 - 10 drops of reagent in roughly 90 minutes. In another test, 100 drops placed in each of 4 wells took just over 10 minutes to complete. While this is an improvement to completely manual test tube methods it is still labor intensive and prone to human error due to the miniscule scale.

The motivation to automate the alignment and reagent deposition process is derived from the small size of the reactors. The potential for contamination and evaporation of a sample became a distraction for the chemical engineering researchers, who needed to focus more exclusively on the scientific questions and problems. While these researchers are certainly capable of automating the system, the time and effort to do so detracts from the problem at hand. Perhaps worse, distractions caused by being burdened with system automation idiosyncrasies have the potential of biasing or misinterpreting experimental results.

Engineering Technology students (Electrical Engineering Technology specifically) were recruited to provide system integration and characterization expertise. These students were selected in part because the EET curriculum focuses on "hands on" application of current and evolving technologies, and thus are well-suited for implementing the tasks of system integration and characterization in a precise and timely manner. Much of this work took place over a summer, so two EET students were recruited, partially to ensure that at least one student was always working on the project to keep it moving. Both students were con- 
sidered equivalent in their strengths and weaknesses regarding technology and interpersonal communication. There roles were by design left fluid. In other words, each was expected to contribute equally to doing whatever it took to get the job done. This meant being equally adept at resolving hardware, software, metrology, and systemic challenges while exchanging ideas and issues on an interpersonal level.

The two EET students interacted on a near daily basis with at first one, then later one additional Chemical Engineering Ph.D. student. The roles of the Chemical Engineering researchers were to communicate system and user interface requirements, working with the EET students to describe and quantify the desired systemic functionality. The Chemical Engineering group was also responsible for equipment procurement and quantitative feedback regarding the usability of the automated system. In addition to the students, faculty members from Chemical Engineering and Electrical and Computer Engineering Technology assumed the roles of mentor and project oversight. Project management was left to the students.

\section{Process Automation and Verification}

Prior to beginning any work, a series of three meetings took place among all parties to get acquainted on technical and interpersonal levels. These meetings consisted of knowledge and documentation exchange, where the Chemical Engineering researchers would describe their goals and provide the EET group with documentation and other equipment specifications. These meetings also provided a forum for exchanging contact information and specifics regarding laboratory location, access and safety. These aspects cannot be over-emphasized due to basic unfamiliarity across disciplines. For example, EET students are much less accustomed to chemical laboratories while Chemical Engineers are less familiar with the ramifications of issues such as insufficient or improper grounding techniques.

The most challenging aspects of automating and characterizing the reagent deposition process involved communication across disciplines whose vocabularies are comparable to any foreign language. Moreover, it was imperative that a computerized automated system be usable by personnel not well versed with computers or the underlying electrical and chemical technologies. Thus it was critical to develop meaningful documentation in the form of user manuals and help screens concurrently with the design, development, and verification of the system. A valuable lesson learned during this project was to anticipate what the next question(s) might be in terms of system capabilities, and try to obtain at least a feel for the solutions during the characterization and verification process.

\subsection{System Integration}

The individual subsystem components used were each of high quality. The system was composed of components produced by five different manufacturers, not to mention the computer 
manufacturer. The motorized x-y stage was produced by Prior Scientific, and the camera by Roper Scientific. A programmable function generator used to control deposition was made by MicroFab, and a Xeon light source manufactured by Sutter Instrument Company. A Nikon Eclipse TE300 Microscope was also utilized for location purposes. Later, as a result of augmented system requirements (another real-world reality), a CCD camera produced by the Photometrics division of Roper Scientific was integrated into the system.

Since each subsystem was built by different companies, their individual control and command API's also varied widely. This challenge was addressed by using a single GUI-based software environment (Visual Basic 6.0) to integrate the subsystems. Each subsystem was first addressed separately, so an "advanced settings" user interface screen was first created for each subsystem separately and then integrated into the user application once characterization and verification was complete. Once each subsystem was integrated into the user application, the automation component of the program could be included. The sequence of events that are needed to take place are composed of three major steps:

1. Movement and location of the $\mathrm{x}-\mathrm{y}$ "stage".

2. Deposition of the reagent into the reactor well.

3. Image capture of the reactor well, capturing the reaction at some point in time.

To arrive at these steps, several informal meetings and discussions were conducted on-the-fly, involving subsets of the interdisciplinary team.

One of the primary challenges of system integration was the initial open-loop nature of the system. It was unclear whether precision could be maintained over a distance of tens or hundreds of wells, even though the $\mathrm{x}-\mathrm{y}$ stage subsystem has a resolution of 1 mum. Another seemingly simple, but initially overlooked aspect of the system was that of a means to immediately terminate the process, an emergency stop feature. While this is generally a common consideration among controls experts it had been completely unaccounted for by others in the group more focused on the science and chemical aspects of the problem. Consideration of this and similar issues underscored some of the advantages of an interdisciplinary team approach.

To begin the user (a lab technician) must "setup" a test by first specifying the sample array size in terms of rows and columns. The team also decided that the user initially locate and align the deposition "jets" over a particular well or group of wells (called "loading"), depending on the number of jets being used (1-4). The layout of the array was a matrix array of rows and columns. The well diameter and their center-to-center spacing is then entered. Moving the sample membrane in a row/column pattern proved to be crucial for maintaining accuracy as shown in figure 2 and discussed later. The technician then also specifies a light filter to be used for each well (a part of the imaging subsystem), time between image captures, number of images to be captured per well, and the volume of reagent (number of drops) to be deposited per well. The user can also precisely control how the reagent material volume is deposited into a well by adjusting a "deposition profile". This profile consists of 
parameters such as open, close, and dwell times. This information is stored and recalled at the correct time during the process.

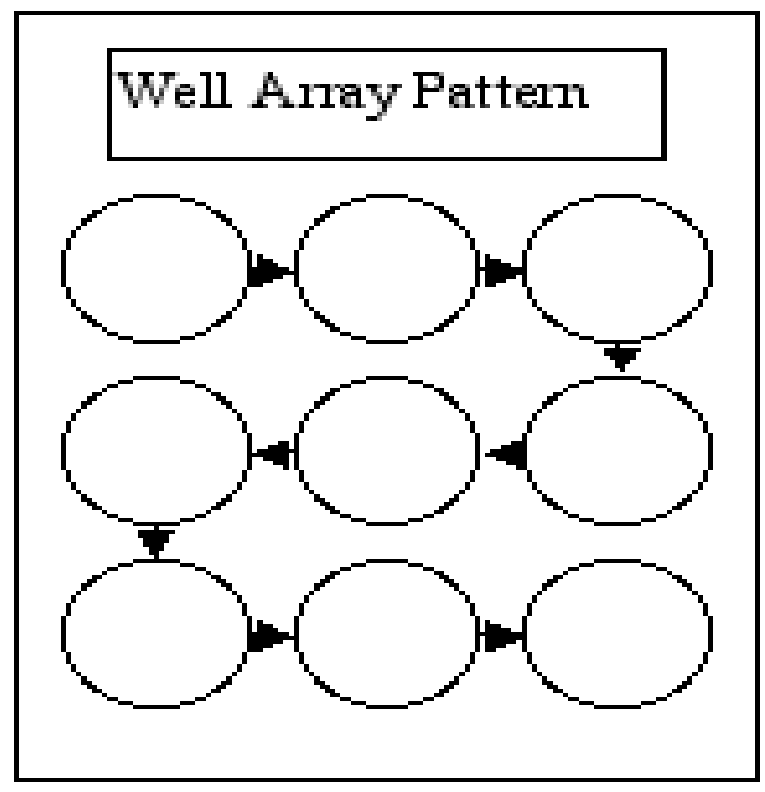

Figure 2: Reactor Well Location Process

Once the initial well locations have been identified and entered the process can begin. The application program positions the stage back to the first well entered using the API specific to controlling the $\mathrm{x}-\mathrm{y}$ stage. Once the stage arrives, the deposition subsystem deposits drops into the well. Again control of the deposition system is performed using the API specific to the deposition subsystem and is transparent to the user. Next the shutter is opened and an image is captured and stored in the imager's native 12-bit raw format of color intensity. Single pixel cross sections, one along the y-axis and the other along the x-axis are stored, dramatically reducing data transfer and storage requirements while maintaining data quality. This helped speed up the program by reducing processing time and allowing for smaller time intervals between pictures. Once the image is stored the camera shutter is closed. If there are no more images to be captured the program automatically moves on to the next well. Thus this method treats each well as an individual experiment.

Visual Basic 6.0 (VB) was selected by the EET group as the means to initially integrate the subsystems. It was chosen for its ease of creating user friendly screen interfaces and built-in I/O port controls. VB 6.0 has many useful built in functions that helped to reduce code length and development time. The built-in serial port control functions were useful because 3 of the 4 pieces of equipment required or could use the serial ports on the computer. The compiler doesn't produce the most efficient code possible, so overall throughput is limited, and is a subject for a future project to ascertain the operational bounds of the 
system using VB. For example, if a timer is used to record the time between two events, the final result will be in error because of a slight overhead delay between when the timer is first initiated and when it actually starts running. This causes timing errors as learned during the project.

An I/O limitation problem surfaced as the motorized stage, light source, and programmable deposition subsystems all required a serial communications port, however the computer used was equipped with only 2 such ports. The team consulted with faculty mentors and decided to connect the motorized $\mathrm{x}-\mathrm{y}$ stage and deposition subsystems to the serial communications ports and use the light source's optional USB connection. The USB interface on the light source's control subsystem was actually a USB to UART interface IC chip. Once all the defined functions from the vendor supplied dynamic link library (.dll) file for the interface chip were installed, operation was simplified and functioned well in the Visual Basic 6.0 environment.

The camera used is a 1392 x 1040-element CCD camera made by Photometrics, a Division of Roper Scientific. The camera interprets each pixel as a 12-bit number and sends it to the computer. Analysis of the chemical reaction compares relative color intensity, not a "visual" intensity as monitored by the human eye. Consultation among group members resulted in a requirement being added that the raw data only needed to be analyzed. This technique resulted in avoiding wasted processing time on creating, formatting and saving an image.

When the data was sent from the camera to the computer it is in a matrix array of numbers that had 1,447,680 entries (1392x1040). Writing these numbers to a simple text file required approximately 5 seconds. This was an unacceptably long amount of time between images as determined by the team. The team arrived at the solution of selecting a single row of pixels near the center of the well in each direction (one row for $\mathrm{x}$ and one column for y), drastically reducing the amount of data required. An example plot of a row of pixels is shown in Figure 3.

\subsection{System Verification}

The first tests performed on the functioning system were intended to test repeatability and accuracy of the $\mathrm{x}-\mathrm{y}$ stage while performing movements. There are two motors on the stage, one for $\mathrm{X}$ direction movements and the other for the $\mathrm{Y}$ direction. Each axis was tested independently to determine if an axis exhibited significant error in the movements and if so, could be corrected in some way. A test program was designed to have the user line up and enter six wells in any pattern. The user would then enter the number of rounds they wanted the program to cycle through the pattern of six. Upon execution, the program would locate each entered well, one at a time, capturing an image of each well. It could be alternatively 


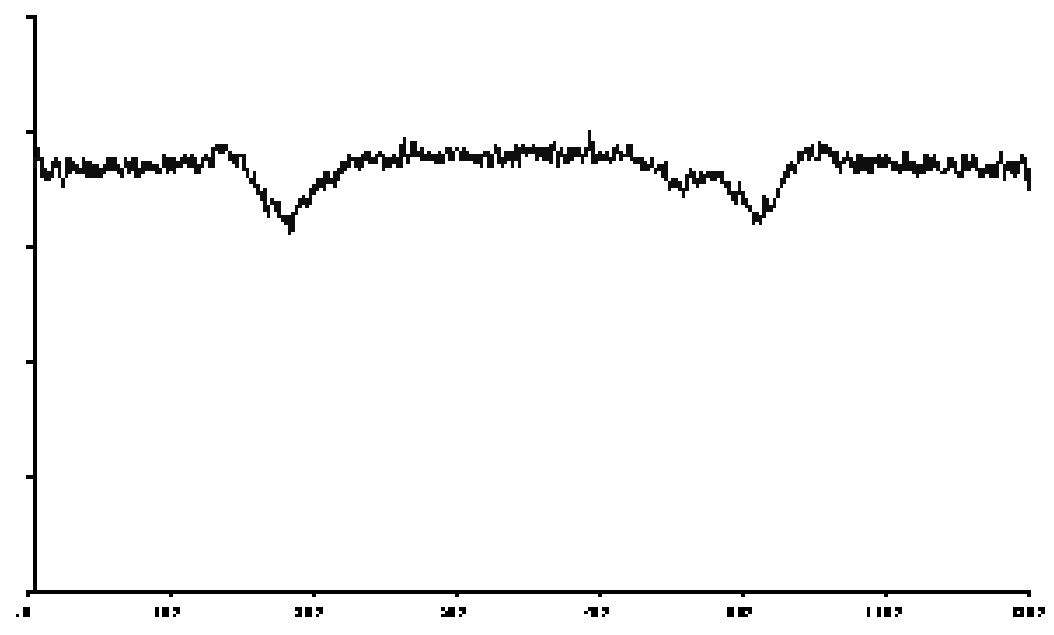

Figure 3: 12-bit color intensity (Y) vs. pixels of a well row (X direction)

specify to capture images after at every movement or just the first round and the last round.

The $\mathrm{x}$-axis tests resulted in an accuracy of $\pm 5 \mu \mathrm{m}$ at $99.7 \%$ confidence over 3600 movements and returning to a specific well 600 times. It was noticed however that the y-axis exhibited significantly more movement error than the $\mathrm{x}$-axis. The error occurred on the first $\mathrm{x}-\mathrm{y}$ movement of our tests. When the stage moved to the first location, it was $\approx 30 \mu \mathrm{m}$ away from its ideal location. After much testing, the cause of the problem was located in the gearing of the stage. When the stage changes direction in the y-axis, the gears require about $30 \mu m$ of distance to lock and start driving in the opposite direction.

To account for this, another (virtual) well location was added to the well array. Now as the first location is loaded, the application program actually loads two locations as illustrated in Figure 4. The first location is set about $300 \mu \mathrm{m}$ above the actual location. When the final well location has been entered and the test has started, the stage returns to the location $300 \mu \mathrm{m}$ above the first well. This allows for the gear to catch as it moves to the first well, and removing the gear slip error for the entire test as all the y-axis movements are down.

It was important to ensure that the camera was tested as well. If the camera itself exhibited a significant error it could add to the any error seen on the stage movements. The camera was tested by initially lining up directly over a single well. Once lined up, the program instructed the camera to capture 600 consecutive images of that well. The data from each image was saved and stored for later analysis in Excel ${ }^{\mathrm{TM}}$. It was initially tested to reproduce use in an actual experiment. This means the actual light source and shutter were used. Once configured, 600 consecutive images were captured of one well. The data 


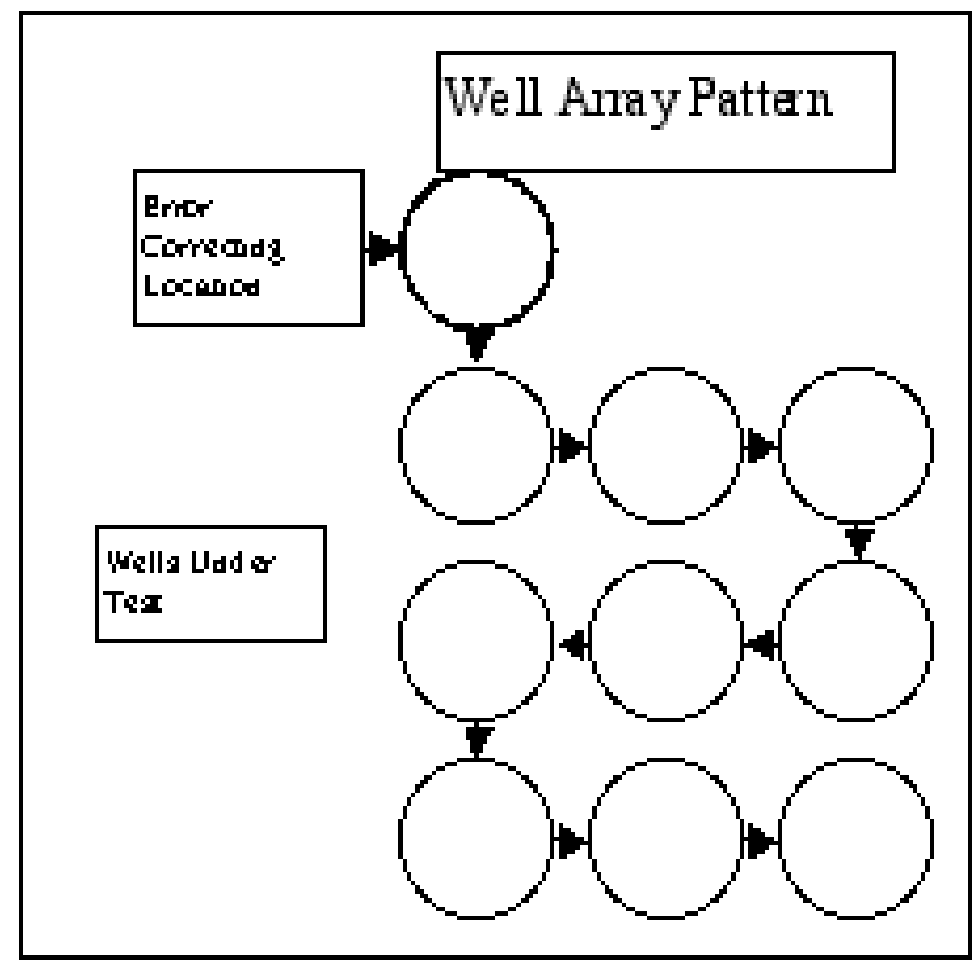

Figure 4: Reactor Well Location Process with Virtual Well Added

was compiled in an Excel spreadsheet, revealing a $\pm 11 \mu m$ error, significantly more than expected. Closer examination showed that the light source shutter was creating vibration in the system just prior to each image capture. The vibration was further characterized in the time and frequency domain, motivating an adjustment to the application program, which was implemented to remove this artifact from the test. The same tests were performed again and the resulting error was within $\pm 2.21 \mu \mathrm{m}$ at $99.7 \%$ confidence. The movable $\mathrm{x}-\mathrm{y}$ stage was then re-tested to re-verify its previous characterization.

\section{Conclusions}

Researchers and students from the fields of Chemical Engineering and Electrical and Computer Engineering Technology collaborated on a six month chemical deposition process automation project. The objective of the project was to integrate several disparate subsystems into an automated system to improve throughput by several orders of magnitude. To this end the project was a success, yet everyone involved gained an appreciation of the challenges of requirements generation, integration, characterization, and verification. Several softer skills were developed and refined under circumstances not found in the classroom, such as project development and troubleshooting completely unknown equipment and software. 
Chemical Engineering students gained in terms of breaking down, then articulating their problem domain to others less accustomed to their terminology. They also observed that it is prudent to address as many contingencies as possible, like providing for an emergency stop feature, as protein and reagent materials are expensive and in some cases physically dangerous. Finally, concepts of procurement and timing were appreciated by all, especially when activities were temporarily delayed due to equipment and parts availability.

Electrical and Computer Engineering Technology students gained a similar appreciation of problem decomposition and communication with other seemingly unrelated technical disciplines. In addition, the students developed skills in contacting technical service personnel from subsystem manufacturers. It was observed that specifications and printed material in user documentation (manuals or Internet sources) rarely agreed with actual practice. Each student member of the EET group learned how to capitalize on personal strengths in order to diminish weaknesses. For example, one student was less experienced using VB, but had a knack for creating user friendly features and functions and designing solutions addressing questions of "what" and "how". Another student was more experienced with implementing basic functionality from limited information and was particularly adept with creating solutions to questions of "where" and "when".

One particular problem encountered involved the open-loop nature of the $\mathrm{x}-\mathrm{y}$ positioning. Course experiences in closed loop control proved valuable in developing a method for closing the loop using the imaging subsystem as the sensing element. Captured images are used for reaction analysis, however the team determined they can also be used for metrology purposes; to "see" and measure distance for well to well dimensional consistency and alignment to the deposition subsystem. One of the most important lessons learned from this experience was in the area of systemic performance tuning. In some cases subsystems required individual tuning while others required innovative means to address subsystem interactions.

Future work includes replication of the automated system so others (biologists and other researchers) can use the system for actual reagent screening. Another area being considered

is to determine the upper bound on throughput, allowing students to participate in the real-world problem of product enhancement and customization.

\section{References}

[1] Gil U. Lee. Purdue proves concept of using nano-materials for drug discovery. Purdue Press, February 2005.

[2] Z. Wang, R.T. Haasch, and G.U. Lee. Mesoporous membrane device for asymmetric biosensing. Langmuir, 21(4):1153-1157, 2005. 